\title{
Collection and Concentration of Light by Touching Spheres: A Transformation Optics Approach
}

\author{
A. I. Fernández-Domínguez, ${ }^{*}$ S. A. Maier, and J. B. Pendry \\ The Blackett Laboratory, Department of Physics, Imperial College London, London SWY $2 A Z$
}

\begin{abstract}
A general three-dimensional transformation optics approach is presented that yields analytical expressions for the relevant electromagnetic magnitudes in plasmonic phenomena at singular geometries. This powerful theoretical tool reveals the broadband response and super-focusing properties of touching metal nanospheres and provides an elegant physical description of the prominent field enhancement that takes place at the point of contact between an spherical nanoparticle and a flat metallic surface.
\end{abstract}

PACS numbers: 78.67.Bf, 73.20.Mf, 42.25.Bs, 42.70.-a

Transformation Optics (TO) was firstly proposed as a strategy to overcome the limitations of computational electromagnetics more than one decade ago [1]. Lately, this theoretical framework has driven the development of metamaterials science, providing a direct link between a desired electromagnetic (EM) phenomenon and the material response required for its occurrence. Thus, during the last years, the guidance of TO [2], together with the advances in fabrication techniques, has made possible the realization of striking EM effects such as optical cloaking. However, the capabilities of TO are not restricted to the design of metamaterial structures. Conformal mapping was already employed in two-dimensional (2D) electrostatics several decades ago [3], and more recently, this subset of transformations have been exploited to study surface plasmon assisted phenomena in 2D geometries [4].

In this letter, we move a step forward in this direction by developing an elegant three-dimensional (3D) TO approach that provides us with a complete physical description of the interaction of light with touching spherical nanoparticles. The optical properties of metal nanostructures in close proximity have been thoroughly analyzed both theoretically [5] and experimentally [6], and studies on the electrostatics of a pair of twin spheres can be found in the literature [7]. However, none of these works have reported the efficient light harvesting capabilities of touching nanoparticle dimers nor the remarkable field enhancement featured by a single nanosphere in contact with a flat metallic surface. Our approach fills this gap. It yields simple analytical expressions for the relevant EM magnitudes in such geometries and evidences the fundamental role that surface plasmon modes play in the highly effective far-to-near field transfer of radiation that occurs in these singular nanoparticle configurations.

Figure 1(a) displays the general structure under study, a dimer of freestanding metallic nanospheres, of radii $R_{1}$ and $R_{2}$ and permittivity $\epsilon_{\mathrm{M}}(\omega)$, that are in contact at a single point. The system is illuminated from the top by a plane wave polarized along the dimer axis ( $z$-direction). We restrict our attention to particle sizes much smaller than the incoming wavelength, which enables us to work (a)

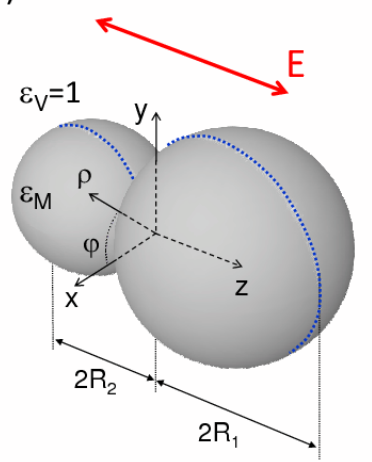

(b)

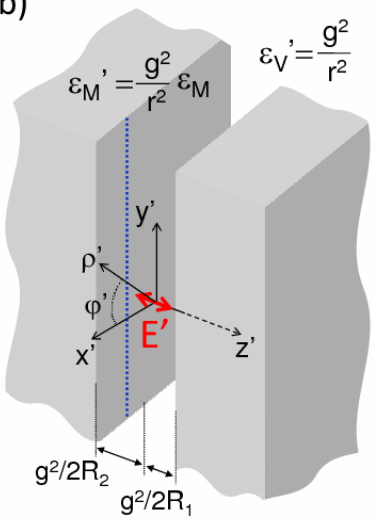

FIG. 1: Panel (a) renders the problem under study: the interaction of light with two metal nanospheres (radii $R_{1}$ and $R_{2}$ ) that touch at a single point. Panel (b) shows the transformed geometry, illumination and permittivities obtained from the inversion of the original system.

within the near-field approximation. Thus, magnetic and electric fields are decoupled, and the latter can be fully described by an electrostatic potential, $\phi(\mathbf{r})$, satisfying Laplace's Equation.

Our solving strategy consists in avoiding treating directly the singular geometry shown in Fig. 1(a) by applying on the system an inversion of the form

$$
\mathbf{r}^{\prime}=\frac{g^{2}}{r^{2}} \mathbf{r}
$$

where primed (unprimed) coordinates correspond to transformed (original) space, and $g$ is an arbitrary length scale constant. Equation (1) transforms the spheres into two semi-infinite slabs. In contrast to $2 \mathrm{D}$ conformal mapping [4], our 3D inversion also acts on the material properties of the system. TO [8] determines that the transformed slabs are not filled with a conventional metal, but with a modified material having a spatially dependent dielectric function of the form $\epsilon_{\mathrm{M}}^{\prime}\left(r^{\prime}, \omega\right)=\frac{g^{2}}{r^{\prime 2}} \epsilon_{\mathrm{M}}(\omega)$, and 
that the relative permittivity between them is $\epsilon_{\mathrm{V}}^{\prime}\left(r^{\prime}\right)=$ $\frac{g^{2}}{r^{\prime 2}}$. Fig. 1(b) renders the geometry and permittivity distribution obtained from the inversion of panel (a).

The illumination, which is described by a uniform electric field along $z$-direction within the near-field approximation, is also transformed under Eq. (1). Its inverted counterpart is given by a potential source of the form $\Phi_{\mathrm{S}}^{\prime}\left(\mathbf{r}^{\prime}\right)=-E_{0} \frac{g^{2}}{r^{\prime 2}} z^{\prime}$, where $E_{0}$ is the amplitude of the original incident field. Importantly, this potential is not a solution of Laplace's Equation. This is a general characteristic of transformed potentials which, due to the spatial dependence of $\epsilon_{\mathrm{M}}^{\prime}$ and $\epsilon_{\mathrm{V}}^{\prime}$, must fulfill

$$
\nabla^{\prime}\left[\frac{1}{r^{\prime 2}} \nabla^{\prime} \Phi^{\prime}\left(\mathbf{r}^{\prime}\right)\right]=0
$$

which expresses Gauss' Law in inverted space. Notice that $\Phi_{\mathrm{S}}^{\prime}\left(\mathbf{r}^{\prime}\right)$ satisfies Equation (2).

The general solution to Eq. (2) can be written as $\Phi^{\prime}\left(\mathbf{r}^{\prime}\right)=r^{\prime} \phi^{\prime}\left(\mathbf{r}^{\prime}\right)$, where $\phi^{\prime}\left(\mathbf{r}^{\prime}\right)$ satisfies Laplace's Equation in transformed space. This sheds light on the physical interpretation of transformed fields and provides us with an expansion basis for $\Phi^{\prime}\left(\mathbf{r}^{\prime}\right)$. Thus, the transformed potential within the dielectric region in Fig. 1(b) can be expressed as the source potential plus a term of the form

$$
\Phi_{\mathrm{V}}^{\prime}\left(\mathbf{r}^{\prime}\right)=\sqrt{\rho^{2}+z^{\prime 2}} \int d k J_{0}\left(k \rho^{\prime}\right)\left[\begin{array}{c}
B^{+}(k) e^{k z^{\prime}}- \\
-B^{-}(k) e^{-k z^{\prime}}
\end{array}\right],
$$

where $\Phi_{\mathrm{V}}^{\prime} \neq \Phi_{\mathrm{V}}^{\prime}\left(\varphi^{\prime}\right)$ due to the azimuthal independence of the source, and $J_{0}$ is the zero-order Bessel function. Equation (3) represents EM modes resulting from the antisymmetric superposition of surface plasmon-like waves that propagate along $\rho^{\prime}$-direction and are confined at the planar interfaces of the inverted geometry. The evanescent tail of these modes within the modified metallic slabs is given by

$$
\Phi_{\mathrm{M}}^{\prime}\left(\mathbf{r}^{\prime}\right)= \pm \sqrt{\rho^{\prime 2}+z^{\prime 2}} \int d k J_{0}\left(k \rho^{\prime}\right) C^{ \pm}(k) e^{\mp k z^{\prime}}
$$

where the upper (lower) sign corresponds to $z^{\prime}>g^{2} / 2 R_{1}$ $\left(z^{\prime}<-g^{2} / 2 R_{2}\right)$. As a result of the expansion procedure, the system unknowns are the coefficients $B^{ \pm}(k)$ and $C^{ \pm}(k)$. These can be obtained by expressing $\Phi_{\mathrm{S}}^{\prime}\left(\mathbf{r}^{\prime}\right)$ in terms of its Hankel transform, and imposing continuity of $D_{z^{\prime}}^{\prime}$ and $E_{\rho^{\prime}}^{\prime}$ at the interfaces of the structure.

Due to the presence of the $r^{\prime}$ prefactor in Eqs. (3) and (4), the matching equations for the transformed fields depend on the parallel coordinate $\rho^{\prime}$. In order to overcome this obstacle, we follow a self-consistent strategy. Since we aim to describe accurately the EM fields behaviour at the contact point of the spheres $(\rho=0)$, we force the continuity of the transformed fields only at its inverted counterpart, $\rho^{\prime} \rightarrow \infty$. Therefore, $D_{z^{\prime}}^{\prime}$ becomes discontinuous at points close to $\rho^{\prime}=0$ within the inverted planar interfaces. We treat the fields mismatch as artificial charge distributions induced at the boundaries of the
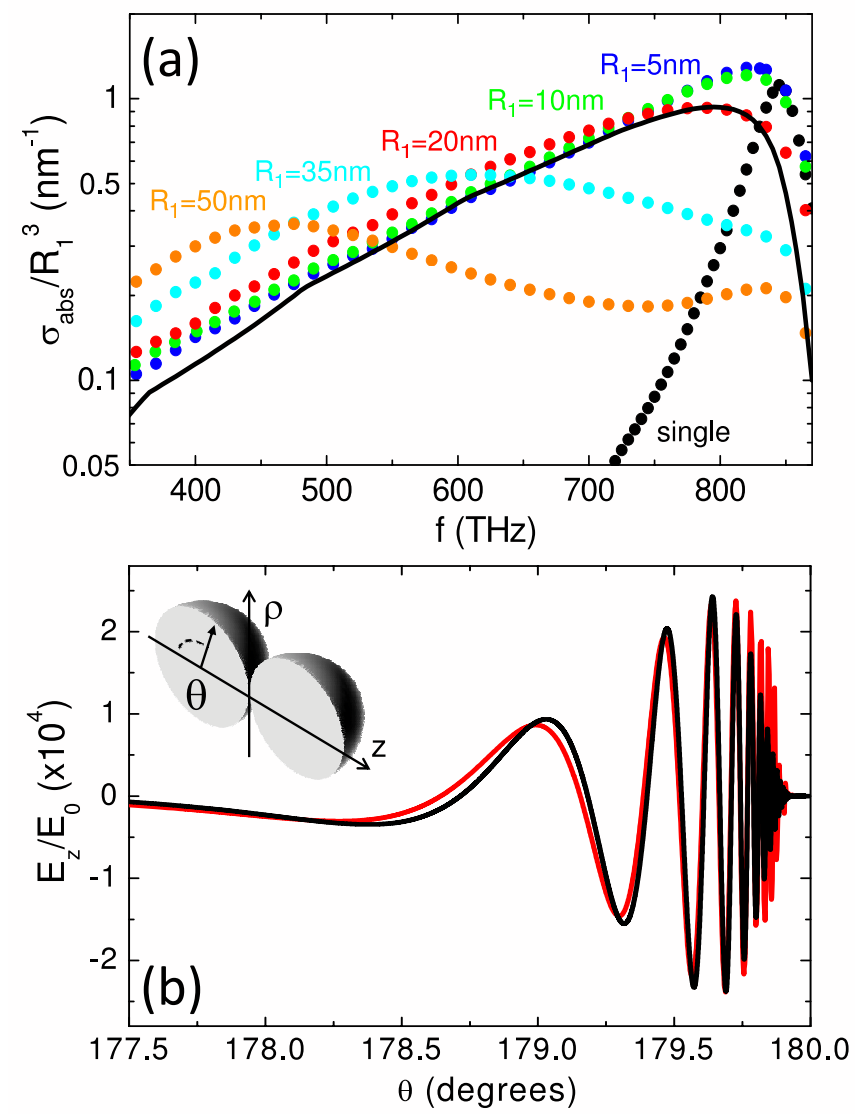

FIG. 2: Panel (a): Numerical absorption cross section, $\sigma_{\text {abs }}$, versus incident frequency, $\mathrm{f}=\omega / 2 \pi$, for twin $\mathrm{Ag}$ touching spheres of different $R_{1}$ (color dots), and a single $5 \mathrm{~nm}$ radius sphere (black dots). Black solid line plots the analytical result obtained from Eq. (5). All the spectra are normalized to $R_{1}^{3}$. Panel (b): electric field amplitude enhancement versus angle $\theta$ (see inset) at $500 \mathrm{THz}\left(R_{1}=20 \mathrm{~nm}\right)$. Black (red) line corresponds to analytical (numerical) calculations.

modified metals, and include them as a correction to our calculations. By keeping only the poles corresponding to the surface waves supported by the structure in the contour integration of Eqs. (3) and (4), and after the inclusion of these surface charge-like corrections, $\Phi^{\prime}\left(\mathbf{r}^{\prime}\right)$ and $\phi(\mathbf{r})=\Phi^{\prime}\left(\mathbf{r}^{\prime}(\mathbf{r})\right)$ are known. Note that, by construction, $\phi(\mathbf{r})$ describes the surface plasmon mediated transfer of EM energy from the incident radiation to the contact point of the spheres.

The dipolar moment induced in the touching spheres by the external fields can be extracted from the far field behaviour of $\phi(\mathbf{r})$. The absorption cross section, $\sigma_{\mathrm{abs}}$, will then be given by the work performed by the illumination on this electric dipole moment, having

$$
\begin{aligned}
\sigma_{\mathrm{abs}} & =\frac{32 \pi^{2} \omega}{3 c}\left(\frac{2 R_{1} \tau}{\tau+1}\right)^{3} \operatorname{Re}\left\{\alpha^{2}\left[1+\cosh \left(\alpha \frac{\tau-1}{\tau+1}\right)\right]-\right. \\
& \left.-\frac{\alpha}{4}(\tau+1)\left[\frac{1}{\tau}\left(1+e^{-\alpha \frac{\tau-1}{\tau+1}}\right)+\left(1+e^{\alpha \frac{\tau-1}{\tau+1}}\right)\right]\right\},(5)
\end{aligned}
$$


where $\alpha=\operatorname{Ln}\left\{\frac{\epsilon_{\mathrm{M}}(\omega)-1}{\epsilon_{\mathrm{M}}(\omega)+1}\right\}, c$ is the velocity of light, and $\tau=R_{2} / R_{1}$ is a parameter that measures the relative size of the two spheres.

Figure 2(a) plots the absorption cross section normalized to the dimer volume for $\mathrm{Ag}[9]$ twin spheres of different sizes calculated using COMSOL multiphysics (color dots). The analytical spectrum obtained from Eq. (5) at $\tau=1$ is also shown in black solid line. The agreement between theory and simulations is remarkable for radii up to $35 \mathrm{~nm}$, where the near-field approximation fails, and radiation losses become significant. Note that the comparison for small $R_{1}$ worsens at large frequencies, as metal absorption prevents EM fields to reach the touching point, where our theoretical results are most accurate. Fig. 2(a) indicates that $\sigma_{\text {abs }}$ for small dimers is of the order of the physical size even at frequencies well below the single sphere resonance (black dots). This demonstrates that touching dimers interact strongly with radiation over the whole optical spectrum, and that their cross section presents a much smoother dependence on frequency than isolated particles.

In order to gain physical insight into the origin of the broadband response of touching spheres, the amplitude enhancement of the $z$-component of the electric field close to the contact point is plotted in Fig. 2(b). Fields are evaluated at $500 \mathrm{THz}\left(\epsilon_{\mathrm{M}}=-16.0+2.1 i\right)$, where the dimer cross section is 50 times larger than the single sphere. For this magnitude, our TO approach yields

$$
\begin{aligned}
\frac{E_{z}}{E_{0}}= & \frac{\pi}{\sqrt{2}(1+\cos \theta)^{\frac{3}{2}}} \times \\
& \times \operatorname{Re}\left\{\alpha^{2}\left(3 e^{\frac{\alpha}{2}}+e^{-\frac{\alpha}{2}}\right) J_{0}\left(\frac{\alpha}{2} \frac{\sin \theta}{1+\cos \theta}\right)\right\},
\end{aligned}
$$

where $\theta$ is defined in the inset of Fig. 2(b). The panel displays the good comparison between theory (black) and simulations for spheres of $20 \mathrm{~nm}$ radius (red). Notice that Eq. (6) does not depend on the dimer size.

Figure $2(\mathrm{~b})$ shows that $\left|\frac{E_{z}}{E_{0}}\right| \geq 10^{4}$ at the vicinity of the contact point of the spheres far from the single particle resonance. This is a clear demonstration of the broadband super-focusing capabilities of the structure. As we have discussed in the outline of our TO approach, this phenomenon is assisted by surface plasmon modes travelling towards the singularity of the structure. EM energy accumulates due to the drastic reduction of group velocity and effective wavelength that the surface waves experience while approaching the contact point of the dimer. Note that neither our TO model nor the numerical simulations include nonlocal effects in $\epsilon_{\mathrm{M}}(\omega)$. However, although these are expected to be relevant below the nanometer scale [10], reductions of only one order of magnitude have been predicted for the intensity enhancement taking place in nanoparticle dimers [11].

Figure 3(a) displays the analytical absorption cross section efficiency, $Q_{\mathrm{abs}}$, for different dimer configurations.
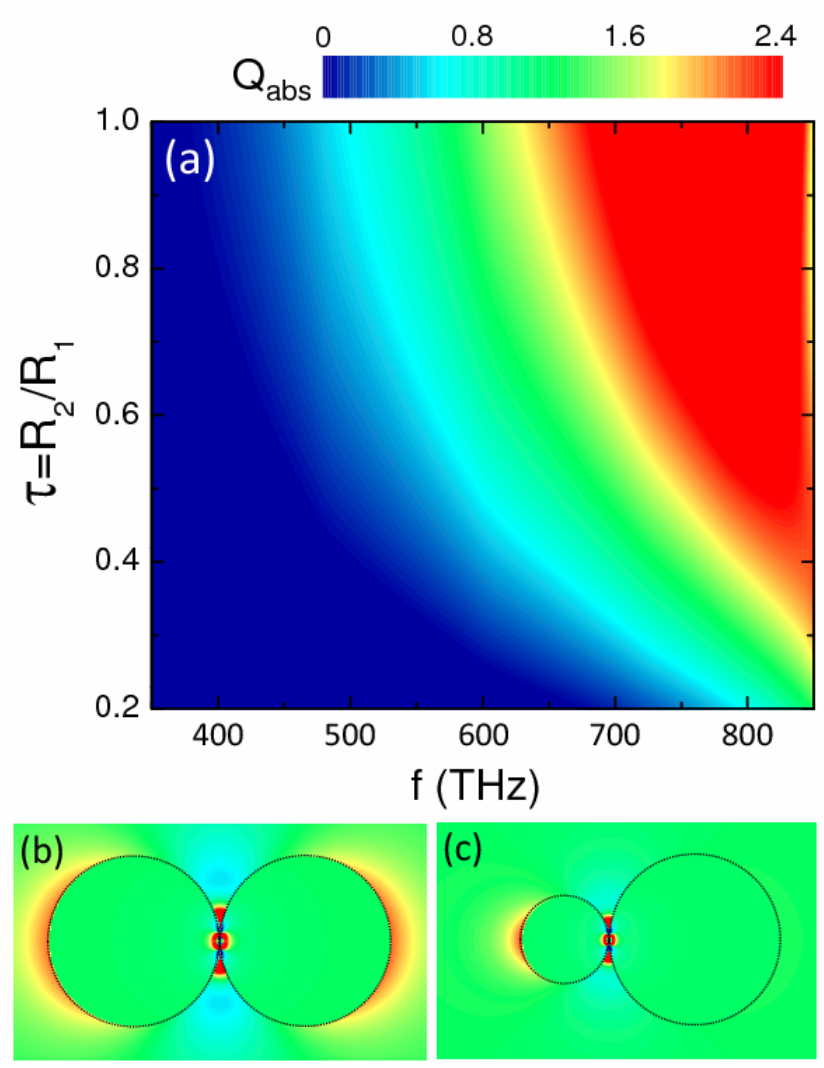

FIG. 3: Panel (a): Absorption cross section efficiency, $Q_{\mathrm{abs}}=$ $\sigma_{\text {abs }} / \pi\left(R_{1}^{2}+R_{2}^{2}\right)$, as a function of the incident frequency and the ratio $\tau=R_{2} / R_{1}$ for $R_{1}=20 \mathrm{~nm}$. Lower panels: Analytical field enhancement at $500 \mathrm{THz}$ within the symmetry plane of the structure for $\tau=1$ (b) and $\tau=0.5$ (c). Saturated colors code $\frac{E_{z}}{E_{0}}$ from -10 (blue) to 10 (red).

The radius of the large sphere is fixed to $R_{1}=20 \mathrm{~nm}$ and the size of the small one ranges from $\tau=0.2$ to 1 . It shows the robustness of the structure properties against geometric asymmetries. The broad frequency region in which $Q_{\text {abs }}>1$ for twin spheres becomes narrower as the size of the small particle is reduced. This sharpening of the spectrum is accompanied by a blue shift of the cross section maximum. Thus, for $\tau=0.2, Q_{\text {abs }}$ presents a narrow peak close to the maximum for the single sphere [see Fig. 2(a)]. Fig. 3(a) shows the transition between the collective response of twin touching spheres to the single particle behaviour that governs the dimer properties when one of the spheres is much smaller than the other. The two lower panels of Fig. 3 depict the analytical $\frac{E_{z}}{E_{0}}$ at $500 \mathrm{THz}$ for $\tau=1$ (b) and $\tau=0.5$ (c). They demonstrate that the strong field concentration at the contact point is preserved in asymmetric dimers, although the focusing efficiency is reduced as $\tau$ decreases.

Finally, we explore another interesting limiting case, $\tau \rightarrow \infty$, which corresponds to a single sphere on top of 

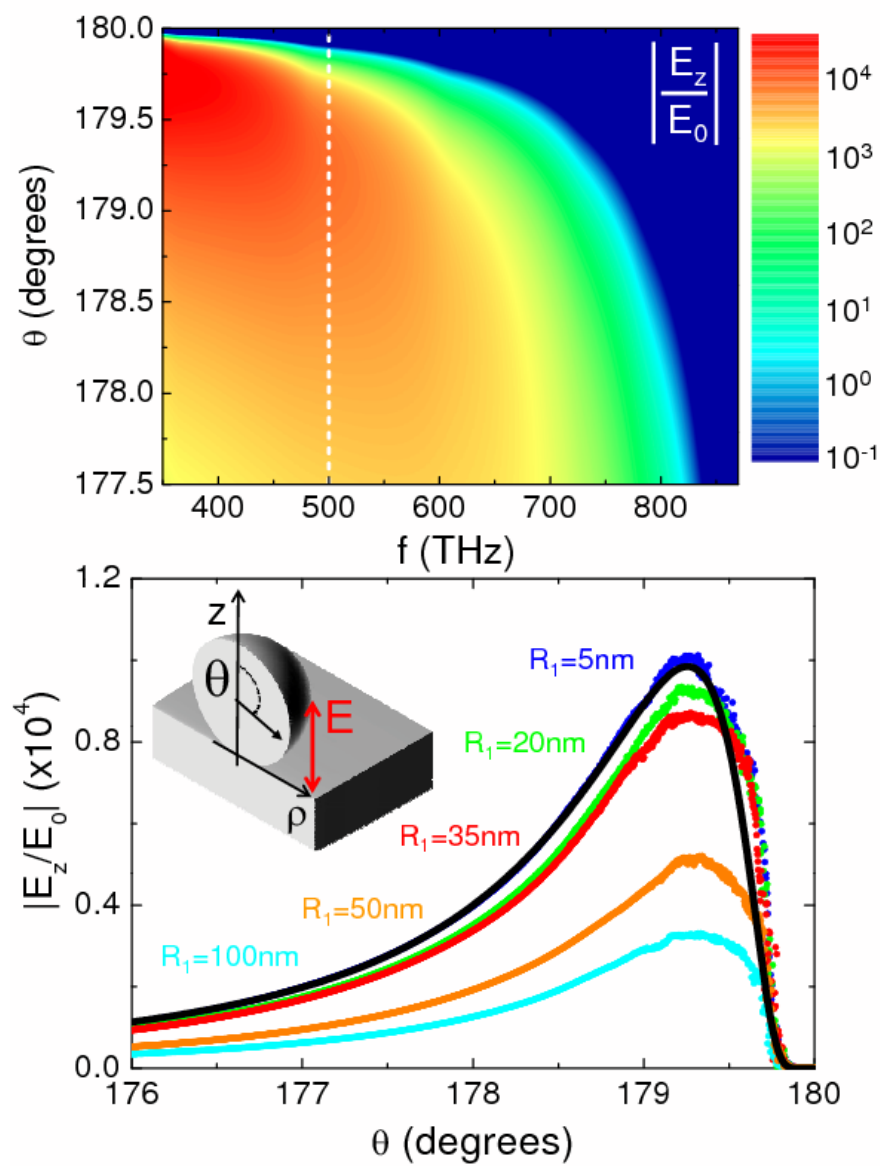

FIG. 4: Upper panel: Analytical absolute value of the electric field enhancement at the touching point between a spherical nanoparticle and a $\mathrm{Ag}$ flat surface as a function of the incident frequency and the angle $\theta$ (see inset). Lower panel: $\left|E_{z} / E_{0}\right|$ for different particle radii at $500 \mathrm{THz}$. Black line plots the analytical result (indicated by dashed white line in the upper panel) and small color dots correspond to simulations.

a metal surface (see inset of Fig. 4). This geometry has demonstrated promising field enhancement capabilities in recent experimental reports [12]. The absolute value of the field enhancement at the structure singularity reads

$$
\left|\frac{E_{z}}{E_{0}}\right|=\frac{8 \sqrt{\pi} \operatorname{Re}\left\{\alpha^{\frac{3}{2}}(1+\cosh \alpha)\right\}}{\sqrt{\sin \theta}(1+\cos \theta)} e^{-\operatorname{Im}\{\alpha\} \frac{\sin \theta}{1+\cos \theta}}
$$

The upper panel of Fig. 4 renders $\left|\frac{E_{z}}{E_{0}}\right|$ as a function of the incident frequency and the angle $\theta$. At low frequencies, fields concentrate at the contact point over a wide angular range. However, the enhancement maximum decreases and departs from the singularity of the structure for larger frequencies. This trend reflects the competition between the two factors in Eq. (7). The former describes the geometric field amplification that occurs when surface plasmon waves are squeezed at the structure singularity. The latter takes into account the drastic increase in damping losses associated with the group velocity reduction experienced by the surface waves at $\theta \sim 180^{\circ}$.

Equation (7) also indicates that the field enhancement featured by a nanosphere placed on top of a metal surface does not depend on the particle size. This result holds only for sphere dimensions much smaller than the wavelength. The lower panel of Fig. 4 displays the comparison between the TO prediction (black line), and full electrodynamic simulations for different particle radii (small color dots) at $500 \mathrm{THz}$. It demonstrates the validity of our approach, and shows that notable amplitude enhancements, of the order of $10^{4}$, occur even for particle diameters comparable to the incoming wavelength.

In conclusion, we have shown that three-dimensional transformation optics can be exploited to study analytically plasmonic effects in geometric singularities. We have demonstrated the highly efficient ability of nanosphere dimers to collect and concentrate broadband radiation at their touching point. We have also evidenced the mechanisms governing the prominent field enhancement that takes place at the point of contact between a spherical nanoparticle and a metal surface. Apart from the profound physical insight that our theoretical results provide on electromagnetic phenomena at singular metal nanostructures, we expect that our analytical approach will make possible a deep exploration of nonlinear and nonlocal effects in such systems.

* Electronic address: a.fernandez-dominguez@ic.ac.uk

[1] A.J. Ward and J.B. Pendry, J. Mod. Opt. 45, 773 (1996).

[2] J. B. Pendry et al., Science 312, 1780 (2006).

[3] R. C. McPhedran et al., Appl. Phys. 24, 311 (1981); A. V. Radchik et al., Phys. Rev. B 46, 6115 (1992).

[4] A. Aubry et al., Nano Lett. 10, 2574 (2010); Y. Luo et al., Nano Lett. 10, 4186 (2010).

[5] D. J. Bergman, Phys. Rev. B 19, 2359 (1979); J. Phys. C 12, 4947 (1979); K. Li et al., Phys. Rev. Lett. 91, 227402 (2003); E. Hao et al., J. Chem. Phys. 120357 (2004); P. Nordlander et al., Nano Lett. 4, 899 (2004); I. Romero et al., Opt. Express 14, 9988 (2006); V. V. Klimov et al., Phys. Rev. B 75, 024303 (2007).

[6] T. Atay et al., Nano Lett. 4, 1627 (2004); M. Danckwerts et al., Phys. Rev. Lett. 98, 026104 (2007); J. B. Lassiter et al., Nano Lett. 8, 1212 (2008); J. A. Fan et al., Science 326, 1135 (2010).

[7] G. S. Smith and R. Barakat, Appl. Sci. Res. 30, 418 (1975); A. V. Paley et al., J. Appl. Phys. 73, 3446 (1993).

[8] D. Schurig et al., Opt. Express 14, 9794 (2006).

[9] E. D. Palik, Handbook of optical constants of solids (Academic Press, New York, 1985).

[10] F. J. García de Abajo, J. Chem. C 112, 17983 (2008).

[11] R. Ruppin, Phys. Rev. B 45, 11209 (1992); J. Zuloaga et al., Nano Lett. 9, 887 (2009); J. M. McMahon et al., Nano Lett. 10, 3473 (2010).

[12] J. J. Mock et al., Nano Lett. 8, 224 (2008); R. T. Hill et al., Nano Lett. 10, 4150 (2010). 